\title{
c-Fos expression in the deep cerebellar nuclei in a rat model of conditioned fear
}

\author{
Véronique M.P. Moers-Hornikx*a,b,d, Johan S.H. Vles ${ }^{b}$, Roelof Jan Hemmes a, Lee Wei Lima,c,d, Govert Hoogland ${ }^{\text {a,c,d }}$, \\ Harry W.M. Steinbusch ${ }^{\mathrm{a}, \mathrm{d}}$, Yasin Temel ${ }^{\mathrm{a}, \mathrm{c}, \mathrm{d}}$
}

\author{
${ }^{a}$ Department of Neuroscience, Maastricht University, Maastricht, The Netherlands \\ ${ }^{b}$ Department of Neurology, Maastricht University Medical Centre, Maastricht, The Netherlands \\ ${ }^{c}$ Department of Neurosurgery, Maastricht University Medical Centre, Maastricht, The Netherlands \\ ${ }^{d}$ European Graduate School of Neuroscience (EURON)
}

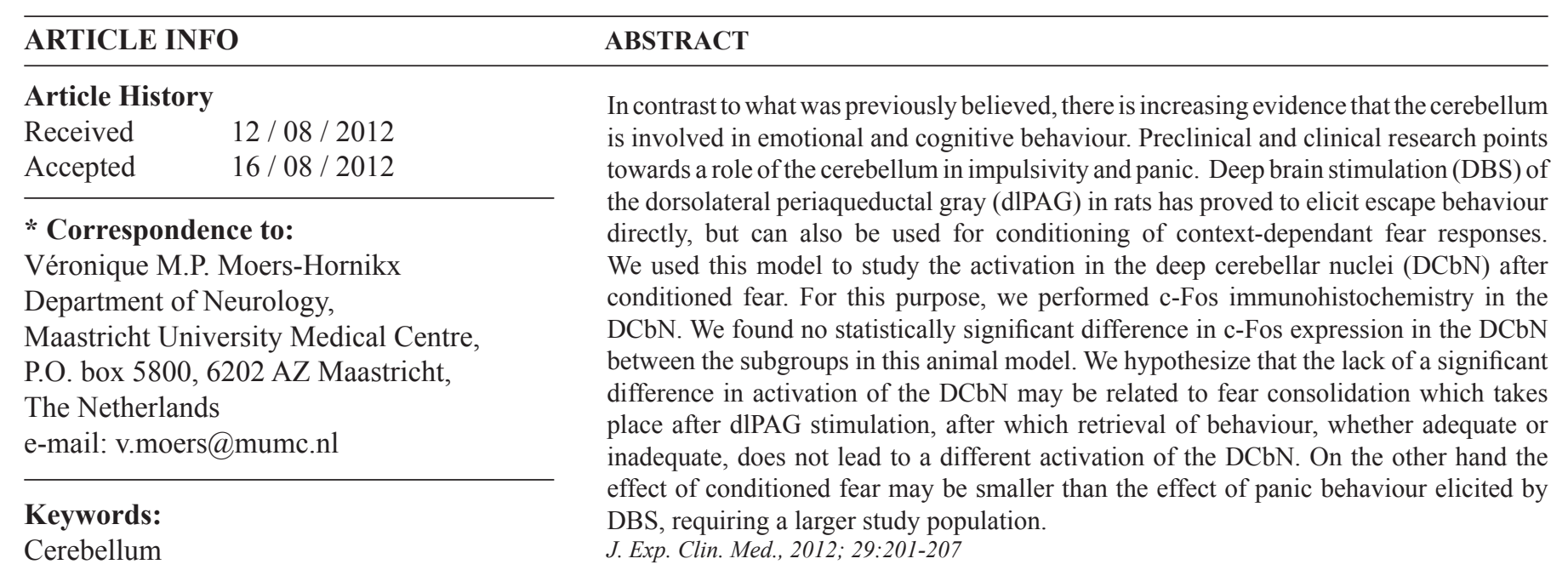

Deep brain stimulation

Periaqueductal grey

Conditioned fear

Escape behaviour

(C) 2012 OMU

\section{Introduction}

The cerebellum has been regarded for decades to be a neuroanatomical structure solely involved in the control of motor coordination (Fine et al., 2002; Manto, 2008; Glickstein et al., 2009; Pearce, 2009). However, there is now more than enough evidence that the cerebellum also plays a prominent role in cognitive and behavioural processes (Leiner et al., 1986; Leiner et al., 1993; Schmahmann and Sherman, 1998). The evolutionary newer regions of the cerebellum project to the prefrontal cortex (areas 9 and 46) and the inferior parietal lobe area 7 through the ventrolateral and other thalamic nuclei including the mediodorsal thalamic nucleus and the reticular nucleus (Yamamoto et al., 1992; Middleton and Strick, 2000; Middleton and Strick, 2001; Cavdar et al., 2002; Allen et al., 2005; Cantalupo and Hopkins, 2010). The cerebellum also receives a wide range of input from structures involved in cognition and affect (Snider and Maiti, 1976; Brodal, 1978; Brodal et al., 1991; Schmahmann and Pandya, 1991; Schmahmann and Pandya, 1993; Dietrichs et al., 1994; Sacchetti et al., 2005; Turner et al., 2007). These struc- tures form the so called cerebrocerebellar circuit for cognition and emotion (Schmahmann, 1996; Allen et al., 2005). Behavioural disturbances resulting from isolated damage to the cerebellum comprise a constellation of symptoms which has been called the cerebellar cognitive affective syndrome (CCAS) (Schmahmann and Sherman, 1998; Marien et al., 2001; Baillieux et al., 2010). These include impairment in executive functions, difficulties with spatial cognition, memory deficiencies, personality changes with disinhibited and inappropriate behaviour, language deficits, and mutism (Paulesu et al., 1993; Schmahmann and Sherman, 1998; Baillieux et al., 2008; de Ribaupierre et al., 2008; Wells et al., 2008; Schmahmann, 2010).

In our previous research we have shown that, in a rat model, impulsivity induced by deep brain stimulation (DBS) of the mediodorsal thalamic nucleus (MD) is associated with deactivation of the deep cerebellar nuclei (DCbN) (MoersHornikx et al., 2009). In rats in which DBS of the dorsolateral periaqueductal grey (dlPAG) induces panic-like behaviour we have shown the same association (Moers-Hornikx et al., 
2011). In contrast rats stimulated in the subthalamic nucleus (STN), known to result in a decrease in impulsivity, show increased activation in the DCbN (Desbonnet et al., 2004; Moers-Hornikx et al., 2011). We hypothesize that the cerebellum plays a role in the selection of relevant information on which a behavioural response is based, thereby coordinating an appropriate response to a stimulus. Deactivation of the cerebellar nuclei may then lead to inappropriate behaviour.

Aside from a role in acute panic, it has been shown that the cerebellum plays a significant role in fear consolidation and fear memory, and therefore also in conditioned fear (Sacchetti et al., 2005; Sacchetti et al., 2009). Lim et al. showed that the dIPAG stimulated rat is not only a good model for acute induced fear- and panic like behaviour, but can also be used for conditioning context dependent fear responses (Lim et al., 2010). In the same study this panic behaviour was also shown to be moderated on a behavioural level by escitalopram, with other research stating that similar antidepressants can reduce fos immunoreactivity in the periaqueductal grey (Lino-deOliveira et al., 2006; Lim et al., 2010). In the present study we performed DBS of the dlPAG in rats to induce conditioned fear. These rats were treated chronically with escitalopram or buspiron. We then determined the activation of the $\mathrm{DCbN}$ as measured by the amount of c-Fos immunoreactivity two hours after a conditioned fear response. As we have previously shown that panic behaviour induced by dlPAG stimulation is accompanied by a decrease of c-Fos expression in the DCbN, we now expected to find a decreased c-Fos expression in the animals showing a conditioned fear response compared to the animals that don't show this conditioned fear response (Lim et al., 2010; Moers-Hornikx et al., 2011).

\section{Materials and methods \\ Subjects}

Male albino Wistar rats $(\mathrm{N}=40,12$ weeks old, bred and housed at the Central Animal Facility of Maastricht University, the Netherlands). Animals were housed individually in standard cages on sawdust bedding in an air ventilated room (temperature approximately $20^{\circ} \mathrm{C}$ ) under a $12 / 12$-h reversed light/dark cycle. Standard laboratory chow (Hopefarms, Woerden, the Netherlands) and water were available ad libitum. At the time of surgery the average body weight of the rats was 300-350 g. The present study was approved by the Animal Experiments and Ethics Committee of Maastricht University.

\section{Experimental groups}

Rats were randomly assigned to one of the following six experimental groups: A. dIPAG stimulation and treatment with saline $(n=7)$; B. dlPAG stimulation and treatment with buspirone $(n=7)$; C. dlPAG stimulation and treatment with escitalopram $(n=7)$; D. dlPAG sham surgery and saline treatment $(n=6)$; E. dlPAG sham surgery and buspirone treatment $(\mathrm{n}=6)$; and F. dlPAG sham surgery and escitalopram treatment $(\mathrm{n}=7)$.

\section{Surgical procedures}

A description of the surgical procedure has been previously reported (Temel et al., 2007; Lim, Blokland et al., 2008; Tan et al., 2010). Rats were anesthetized using ketamine $(90 \mathrm{mg} /$ $\mathrm{kg}$ ) and xylazine $(10 \mathrm{mg} / \mathrm{kg}$ ) injected subcutaneously (s.c.). By means of a stereotactic apparatus (Stoelting, Wood Dale,
USA; model 51653) burr holes were made in the skull through which a gold-plated electrode with inner platinum- iridium combination was implanted (Technomed, Beek, the Netherlands) at the level of the right dlPAG (coordinates from Bregma: anteroposterior,-7.6 mm; mediolateral, +0.7 $\mathrm{mm}$; and ventral, $-4.8 \mathrm{~mm}$; approached with a coronal angle of $10^{\circ}$; Paxinos and Watson, 1998). The electrodes were fixed in position using dental cement (Heraeus Kulzer, Hanau, Germany). After surgery the rats were injected with Temgesic $(0.1 \mathrm{mg} / \mathrm{kg}$, s.c. $)$ for analgesia and allowed to recover for a period of two weeks.

\section{Deep brain stimulation}

Stimulation was performed at a frequency of $50 \mathrm{~Hz}$ and pulse width of $0.1 \mathrm{~ms}$ based on previous experience (Lim et al., 2010). The amplitude was gradually increased until escape behaviour was observed. Stimulation at each amplitude was performed during $15 \mathrm{~s}$ followed by a stimulation-off period of $45 \mathrm{~s}$. Two consecutive positive responses of escape reaction were required to determine the escape threshold. The mean amplitude neccesary to elicit an escape response was $60 \mu \mathrm{A}$. Animals which required stimulation intensities above $100 \mu \mathrm{A}$, suggesting incorrect positioning of the electrode, were discarded from analysis. A World Precision Instruments (WPI) digital stimulator (DS8000, WPI, Berlin, Germany) and a stimulus isolator (DLS100, WPI, Berlin, Germany) were used to deliver the stimuli. After the threshold determination session, all rats were given a recovery period of two weeks. The sham animals were similarly connected to the stimulator but no current was delivered to the dlPAG.

\section{Drug administration}

Escitalopram oxalate (H. Lundbeck A/S, Copenhagen, Denmark) and buspirone hydrochloride (TOCRIS, Cookson Inc., Missouri,USA) were dissolved in saline $0.9 \% \mathrm{NaCl}$, as described previously (Lim et al., 2010). Habituation to the injection procedure was obtained by injection of $1 \mathrm{ml}$ saline three times on alternating days one week before the actual experimental testing. The animals received 21 daily injections of either escitalopram (ESCIT, s.c., $10 \mathrm{mg} / \mathrm{ml}, 1 \mathrm{ml} / \mathrm{kg}$ ), buspiron (BUSP, s.c., $3 \mathrm{mg} / \mathrm{ml}, 1 \mathrm{ml} / \mathrm{kg}$ ), or saline (SAL, $1 \mathrm{ml} / \mathrm{kg}$ ), respectively. Chronic treatment was chosen as the maximal effect of these drugs is expected in the chronic phase (Burghardt et al., 2004; Assie et al., 2006; Bondi et al., 2008; Lim et al., 2008; Sato et al., 2008; Lim et al., 2010). The final drug administration was performed $60 \mathrm{~min}$ (ESCIT, SAL) or 120 $\min$ (BUSP) before the test in the open-field.

\section{Behavioural testing}

Rats were tested in an open field (OF). Details of this test were published previously by Lim et al (Lim et al., 2010). The OF consisted of a clear Plexiglas box (square: $100 \mathrm{~cm}$ $\mathrm{x} 100 \mathrm{~cm}$, and height: $40 \mathrm{~cm}$ ) with open top and dark floor (Lim et al., 2008). All open-field testing was conducted in the same room in a dimly lit condition. On the day of stimulation in the open-field, rats were placed in the open-field arena and connected with the external stimulator through externalized leads followed after 1 minute by electrical stimulation with parameters based on the previously determined escape threshold. Once the escape reaction was evoked, the cable connected to the rat was detached and the animal was left in the 
open-field for approximately $30 \mathrm{~s}$. The electrical stimulation to elicit an escape reaction was always carried out between 20:00 and 22:00 h.

Twelve hours after the induced escape behaviour (between 08:00 and 10:00 h) animals were placed in the OF again during which the behaviour of each rat was recorded using an automated system consisting of a camera connected to a computer with the Ethovision tracking software (Ethovision, Noldus Information Technology, Wageningen, the Netherlands). The Ethovision software automatically calculated and analyzed temporospatial data of the animal relative to the OF including locomotion/ distance moved, velocity and time spent in the corner of the open field, each parameter on a per 2 minutes basis. A trial was stopped after 5 minutes and the rat was removed from the arena. Fear or freezing behaviour was defined by a decreased locomotion (immobility) and increased duration of time spent in the corner of the OF. Escape behaviour was characterized by rigorous and aimless running within the OF arena.

\section{Histological processing}

Two hours after the final testing of the rats in the OF they were placed under generalized anaesthesia with Nembutal $(75 \mathrm{mg} / \mathrm{kg})$ and transcardially perfused with Tyrode $(0.1 \mathrm{M})$ and a fixative solution which consists of paraformaldehyde, picric acid and glutaraldehyde in phosphate buffer ( $\mathrm{pH} 7.6)$. After two hours of post fixation the brains were cryoprotected by overnight sucrose and then quickly frozen with $\mathrm{CO}_{2}$ and stored at $-80^{\circ} \mathrm{C}$. One series of brainstem sections per animal was stained with standard hematoxylin-eosin (Merck, Darmstadt, Germany) to examine the localization of the electrode tips.

The cerebelli were cut serially on a cryostat (MICROM, Walldorf, Germany) into $10 \mu \mathrm{m}$ frontal sections which were collected on gelatin-coated slides and then stored at $-30^{\circ} \mathrm{C}$. We used a previously published protocol for c-Fos immunohistochemistry with minor modifications (Moers-Hornikx et al., 2011). In short, we used a primary rabbit polyclonal $\mathrm{IgG}$ antibody against c-Fos (diluted 1:300, Santa Cruz Biotechnology Inc., Santa Cruz, CA, USA) for incubation at room temperature for two days on a constant shaker. Subsequently sections were incubated with the secondary antibody (diluted 1:400, biotinylated donkey anti- rabbit biotin; Jackson Immunoresearch laboratories Inc., Westgrove, USA) overnight. This was followed by incubation for 2 hours with an avidinbiotin-peroxidase complex (diluted 1:800, Elite ABC-kit, Vectastatin; Vector, Burlingame, USA). To visualize the immune complex of horseradish peroxide (HRP) reaction product, the sections were incubated with 3,3'-diaminobenzidine tetrahydrochloride (DAB)/nickel chloride $\left(\mathrm{NiCl}_{2}\right)$ solution. Finally sections were dehydrated and cover slipped with Pertex (HistolabProducts, Goteborg, Sweden).

A Nissl stain was conducted on a parallel series of 10 $\mu \mathrm{m}$ sections to facilitate delineation of the boundaries of the $\mathrm{DCbN}$. This parallel series was first air-dried for 30 minutes and subsequently exposed to a buffer solution (40 mL 136.08 $\mathrm{g} / \mathrm{mol}$ sodium acetate with $9.6 \mathrm{ml}$ glacial acetic acid in a 1000 $\mathrm{ml}$ distilled water solution (milli-q)), followed by a $2 \%$ triton $\mathrm{x}-100$ solution of $2.5 \mathrm{ml}$ with $150 \mathrm{ml}$ absolute alcohol and 50 $\mathrm{ml}$ distilled water, then the previously described buffer solution again, and finally, a Cresyl violet solution $(0.1 \mathrm{~g} / 1000 \mathrm{ml}$ distilled water). Finally sections were rinsed with the buffer solution, dehydrated, and cover slipped with Pertex (HiStolabProducts, Goteborg, Sweden).

\section{Quantative evaluation of c-Fos immunoreactive cells}

Systematic cell counts of c-Fos immunoreactive (c-Fos-ir) cells were performed in the $\mathrm{DCbN}$ as previously described (Moers-Hornikx et al., 2011). Photography of both the c-Fos and parallel Nissl stained sections was performed at a $4 \mathrm{X}$ magnification on an Olympus AX70 bright-field microscope with an Olympus DP70 camera (analySIS; Imaging System, Münster, Germany). The bregma levels of the DCbN ranged from -10.52 to $-11.80 \mathrm{~mm}$, which was verified with the Paxinos and Watson Atlas of 1998 (Watson, 1998).

In the c-Fos stained sections the delineation of the $\mathrm{DCbN}$ was difficult due to a weak background staining. As mentioned above, a parallel series of sections was stained with a Nissl-staining to aid in delineation. Using Image $\mathrm{J}$ (version 1.43), a digital substraction of the $\mathrm{DCbN}$ as delineated in the Nissl stained sections were projected on the c-Fos stained slides thereby giving a more accurate delineation of the cerebellar nuclei. Minor deviations in this technique due to slight difference in bregma level were corrected for manually. Artefacts in the sections were excluded from analysis to ensure accuracy of measurements. A cell was defined as being c-Fos-immunoreactive (c-Fos-ir) if it had a 65\% higher density than the corresponding background grey value of the $\mathrm{DCbN}$. The number of c-Fos-ir cells per $\mathrm{mm}^{2}$ was calculated for each nucleus.

\section{Statistical analysis}

Since we were primarily interested in the effect of conditioned fear on c-Fos expression in the $\mathrm{DCbN}$, the treatment groups were pooled according to whether they did or did not show escape behaviour in the final test in the OF. The data of the c-Fos counts were analysed using the Mann-Whitney test for non-parametric data (Monte Carlo method) since the group sizes were relatively small and non-normally distributed. The significance level was set at $p<0.05$. Furthermore we performed a Kruskall-Wallis test to analyse differences between stimulated and sham stimulated groups. Post hoc analysis to follow up significant findings from the Kruskal-Wallis test was performed using the Mann-Whitney test with a one-tailed procedure. The level of significance for the Kruskal-Wallis test was set at $\mathrm{p}<0.05$, and for the post-hoc Mann-Whitney tests it was set at $p<0.0167$. Output is provided as the number of c-Fos ir cells per square millimeter with 2 standard error of the mean values (S.E.M).

Any outliers identified in the c-Fos data on a per rat per nucleus basis were corrected for by transforming the data into the maximal 2SD+/- the mean. Rats were excluded if their total average values exceeded the $2 \mathrm{SD}+/-$ the mean for the total of the groups of rats. Finally 12 rats showing freezing behaviour and 24 rats showing no freezing behaviour were included for statsitical analysis.

To determine the amount of agreement concerning the semi-quantitative method of cell counting between RJH and $\mathrm{VMH}$ an intraclass correlation coefficient (ICC) was determined on previously stained c-Fos DCbN. This was done using the Statistical Package for the Social Sciences software (SPSS, version 17.0.2, 11th march 2009) in a two- way- mi- 
xed model of the exact type.

\section{Results}

\section{Electrode localization}

The histological evaluation of the electrode localization was previously described by Lim et al. (Lim et al., 2010). The electrode tip was correctly placed in the dlPAG in six animals per group for the sham-saline, sham-buspirone, stimulationsaline and stimulation-escitalopram groups and seven animals for the stimulation-buspirone and sham-escitalopram group (Lim et al., 2010).

\section{Behavioural results}

Results of behavioural testing were published previously by Lim et al (Lim et al., 2010). In summary electrical stimulation of the dIPAG evoked escape behaviour which was followed by decreased locomotion or a freezing response when animals were placed back in the OF 12 hours later; a behavioural pattern indicative of conditioned fear.

Escitalopram reduced the time in the corner squares of the OF in the stimulated animals but not in the sham animals $(F(2.33)=10.12, p<0.01)$. Stimulation decreased the mean velocity of movements $\mathrm{F}(1.33)=16.57, \mathrm{P}<0.01$ ) while escitalopram corrected for this in the stimulated animals but not the sham animals.

\section{Quantification of c-Fos-ir cells}

There were no significant differences between the left and right $\mathrm{DCbN}$, therefore the results from the left and right hemispheres were pooled for further analysis. There were no significant differences in c-Fos expression in the DCbN between rats that showed conditioned fear in the final OF session and those that did not. Furthermore there were no significant differences in c-Fos expression in the $\mathrm{DCbN}$ between the treatment groups. (Fig. 1-3).

A few cells displayed a hyperintense cytoplasma. These were not considered to be the 'classically' stained c-Fos ir cells as often described in the literature and therefore not included in the data analysis.

\section{Intraclass correlation coefficient}

The intraclass correlation coefficient was determined based on the total c-Fos ir cells $/ \mathrm{mm}^{2}$ counted by RJH in comparison to $\mathrm{VMH}$ for all the $\mathrm{DCbN}$ in a previous experiment. The ICC for the total of the $\mathrm{DCbN}$ yielded a correlation of 0.795 (single value; 0.886 for average), which is a substantial to high level of agreement on the Landis \& Koch scale.

\section{Discussion}

In the present study we analysed changes in activation in the deep cerebellar nuclei after a conditioned fear response 12 hours after dlPAG-stimulation. We expected a decrease in activation in the animals which show this conditioned fear response in comparison to sham stimulated animals. In the animals treated with escitalopram which do not show a conditioned fear response after dlPAG stimulation we expected a normalisation of the deactivation of the $\mathrm{DCbN}$. We were however not able to show a difference in activation in the $\mathrm{DCbN}$.

The amygdala and the hippocampal complex are important structures in fear memory (Sacchetti et al., 2005). There

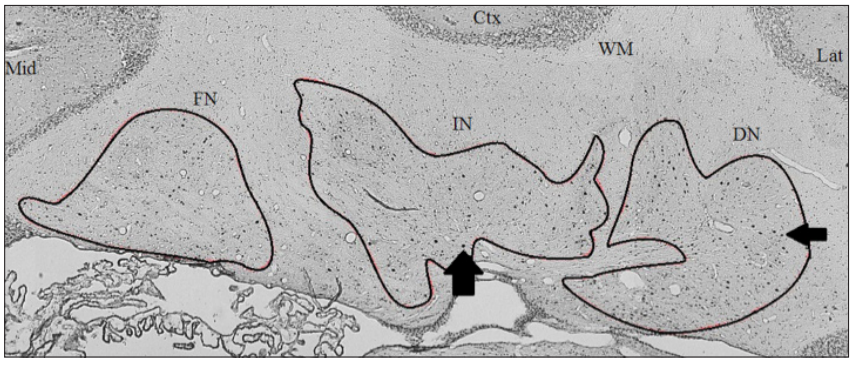

Fig. 1. Representative low-power photomicrograph of a $10 \mu \mathrm{m}$ thick coronal section of the right cerebellar hemisphere at approximately bregma level $-11.70 \mathrm{~mm}$ (in transition from $-11.60 \mathrm{~mm}$ to $-11.80 \mathrm{~mm}$ ) showing c-Fos-ir cells in the $\mathrm{DCbN}$. The $\mathrm{DCbN}$ are delineated by the black lines. The black arrows point towards a c-Fos positive cell. Abbreviations: $4 \mathrm{~V}=$ fourth ventricle; $\mathrm{Ctx}=$ cerebellar cortex; $\mathrm{DN}=$ dentate nucleus; $\mathrm{FN}=$ fastigial nucleus; $\mathrm{IN}=$ interposite nucleus; Lat=lateral aspect of cerebellar hemisphere; Mid=midline; $\mathrm{Pc}=$ choroid plexus; $\mathrm{Wm}=$ white matter

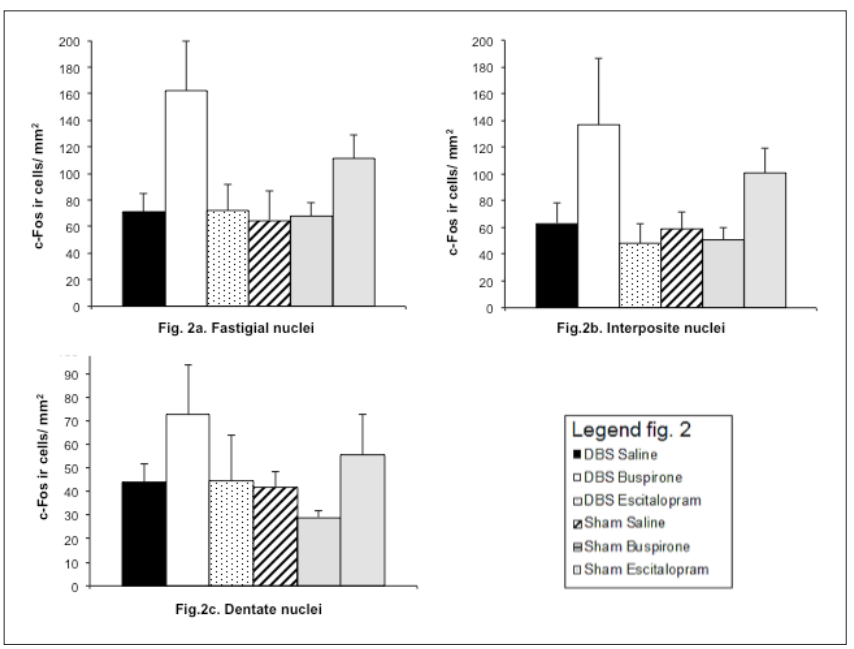

Fig. 2. C-Fos expression in the deep cerebellar nuclei. Data are presented as mean plus S.E.M. showing six treatment groups: animals stimulated in the dorsolateral periaqueductal gray (dlPAG) and treated with respectively saline, buspiron or escitalopram, followed by animals with sham dIPAG stimulation again treated with respectively saline, buspiron or escitalopram. Data are presented for the fastigial nucleus of the cerebellum (FN; Fig. 2a), the interposite nucleus (IN; Fig. 2b) and the dentate nucleus (DN; Fig. 2c). Note the higher density of c-Fos-ir cells in the dlPAG-buspiron group and in the sham-dlPAG-escitalopram group which is consistently present in all nuclei, however, without reaching significance.

is also evidence for a substantial role of the cerebellum in the process of fear consolidation and retrieval. A key structure is the Purkinje cell (PC). Pairing of an unconditioned stimulus and a conditioning stimulus leads to long term depression (LTD) of the parallel fibre synapses with the PC's. This decreases the firing rate of the PC's, which allows the conditioned stimulus to be passed on to the DCbN (Sacchetti et al., 2005; Sacchetti et al., 2009). Fear conditioning is important for survival, therefore fear memory is rapidly acquired and long lasting (Sacchetti et al., 2005). In classical Pavlovian conditioning several training sessions are usually necessary to elicit a conditioned fear response. In our study a short stimulation of the dIPAG in the open field (OF) is enough to elicit a conditioned fear response when rats are placed in the same OF arena 12 hours later (Lim et al., 2010). Possibly this strong 


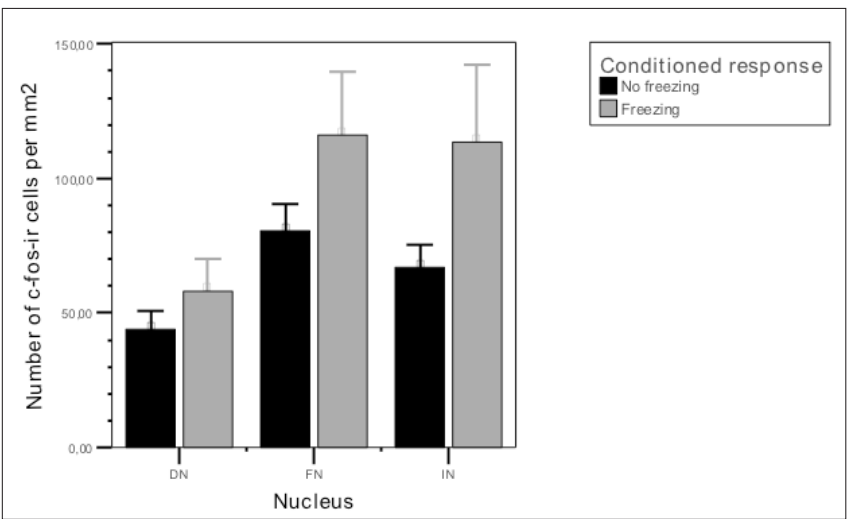

Fig. 3. C-Fos expression in the deep cerebellar nuclei. Data are presented as mean plus S.E.M. showing the pooled treatment groups: animals showing freezing behaviour 12 hours after dorsolateral periaqueductal grey (dIPAG) stimulation and animals that don't show this freezing behaviour. Data are presented for the dentate nucleus $(\mathrm{DN})$, the interposite nucleus (IN) and the fastigial nucleus (FN).

stimulus already causes synaptical changes in the cerebellum. One could then hypothesize that in the rats with a conditioned fear response, retrieval of this conditioned behaviour does not cause a different activation than retrieval of normal behaviour in the non-conditioned rats or in the rats where the consolidation of this fear memory has been inhibited by treatment with escitalopram. This may account for our not finding any difference in activation in the $\mathrm{DCbN}$ between the different groups.

A different explanation is related to the stimuli themselves. In a previous study we found a decreased activation in the DCbN directly after panic behaviour caused by dIPAGstimulation (Moers-Hornikx et al., 2011). In the present study the delay between stimulation and sacrifice was 14 hours, in contrast to two hours in the previous study. Therefore we did not expect to see an effect of stimulation since the maximal expression of c-Fos takes place two to four hours after a stimulus (Smith et al., 1992). Electrical stimulation is a very strong stimulus. Possibly the conditioned fear is a weaker stimulus, as a result of which group sizes may not have been adequate to disclose a significant effect. This may furthermore account for the low and variable signal intensity seen in the sections. The variability in signal intensity was not seen in the cerebellar cortical granule cell layer, therefore it could not be attributed to mere staining quality. There was no significant lateralization across the different treatment groups. This could be related to the suboptimal expression. It is also likely that the effects of dlPAG-stimulation are not confined to just one of the cerebellar hemispheres but have a more generalized effect (as claimed in the DBS background effect theory), since many structures in the cerebrocerebellar loop are (at least partially) bilaterally interconnected (Allen et al., 2005).

Although not significant, there is a substantial increase in the amount of c-Fos ir $/ \mathrm{mm}^{2}$ in the DBS dlPAG- buspirone group compared to the sham-buspirone group (from 50 cells $/ \mathrm{mm}^{2}$ in the sham buspirone group to 124 cells $/ \mathrm{mm}^{2}$ in the dlPAG- buspirone group) and a decrease in the amount of c-Fos ir $/ \mathrm{mm}^{2}$ in the dlPAG-escitalopram group compared to the sham-escitalopram group (from 68 cells $/ \mathrm{mm}^{2}$ in the sham-escitalopram group to 51 cells $/ \mathrm{mm}^{2}$ in the dlPAGescitalopram group) (Fig. 2). These changes did not show a correlation with the measured behavioural parameters: freezing and escape behaviour increased in the dIPAG stimulated group as a whole and this effect was normalised only by escitalopram in the stimulated group. A possible explanation could be that there is a mild combined permissive effect of DBS with buspirone treatment on c-Fos expression without any significant change in the measured behavioural parameters. No evidence was found in literature to back up this hypothesis.

In some of the sections we identified hyperintense cytoplasma in the absence of any nuclear staining with c-Fos. This was independent of the intensity of the staining. Some of these cells showed almost isointensity with adjacent c-Fos stained nuclei. This could be due to Fos-like immunoreactivity (Fos-LI) as has previously been described (Duan et al., 1999). Possibly there is an influence of deep brain stimulation induced electrochemical changes, altering electrical charges or even inducing cellular alterations considering the trafficking of proteins from diverse intracellular departments. These sparsely distributed cells were seen in only a few sections of the dIPAG stimulated group with escitalopram and sham with saline and escitalopram. Therefore there was no relation with the treatment group, which pleads against an influence of DBS. The number of these cells was too small to perform statistical analysis on. However, a vue microscopy did show that these cells were more often to be found in the lateral regions of the $\mathrm{DCbN}$. This interesting finding could point towards several things. It could indicate that these cells are of a different subset of neurons or that the functional state of these neurons has changed (i.e. different metabolism or a pre-apoptotic state). The exact nature of this phenomenon remains a topic of discussion.

\section{Conclusion}

In this study we found no significant change in activation of the $\mathrm{DCbN}$ in rats that showed a conditioned fear response induced by dIPAG stimulation 12 hours before testing. We hypothesize that the lack of a significant difference in activation of the $\mathrm{DCbN}$ may be related to fear consolidation which takes place after dIPAG stimulation, after which retrieval of behaviour, whether adequate or inadequate, does not lead to a different activation of the DCbN. In our previous research we found deactivation of the $\mathrm{DCbN}$ associated with panic behaviour induced by dIPAG-stimulation. The conditioned fear response may have caused smaller changes in cerebellar activation which were not disclosed due to small group sizes.

\section{Coflict of interests}

The authors declare that they have no conflict of interests.

\section{Acknowledgements}

The authors are thankful to $\mathrm{H}$. Lundbeck A/S for the donation of escitalopram oxalate. The behavioural research was supported by a grant from the FP6 Marie Curie Fellowship (MEST-CT-2005-020589) and by a grant from the Netherlands Organization for Scientific Research (NWOVeni,No: 016.096.032).

The authors are grateful to Mrs Helen Steinbusch and Ms Marjan Phillipens for their technical assistance. 


\section{REFERENCES}

Allen, G., McColl, R., Barnard, H., Ringe, W.K., Fleckenstein, J., Cullum, C.M., 2005. Magnetic resonance imaging of cerebellar-prefrontal and cerebellar-parietal functional connectivity. NeuroImage. 28, 39-48.

Assié, M.B., Lomenech, H., Ravailhe, V., Faucillon, V., Newman-Tancredi, A., 2006. Rapid desensitization of somatodendritic 5-HT1A receptors by chronic administration of the high-efficacy 5-HT1A agonist, F13714: A microdialysis study in the rat. Br. J. Pharmacol. 149, $170-178$.

Baillieux, H., De Smet, H.J., Dobbeleir, A., Paquier, P.F., De Deyn, P.P., Mariën, P., 2010. Cognitive and affective disturbances following focal cerebellar damage in adults: A neuropsychological and SPECT study. Cortex. 46, 869-879.

Baillieux, H., De Smet, H.J., Paquier, P.F., De Deyn, P.P., Mariën, P., 2008. Cerebellar neurocognition: Insights into the bottom of the brain. Clin. Neurol. Neurosur. 110, 763-773.

Bondi, C.O., Rodriguez, G., Gould, G.G., Frazer, A., Morilak, D.A., 2008. Chronic unpredictable stress induces a cognitive deficit and anxietylike behavior in rats that is prevented by chronic antidepressant drug treatment. Neuropsychopharmacol. 33, 320-331.

Brodal, P., 1978. The corticopontine projection in the rhesus monkey. Origin and principles of organization. Brain. 101, 251-283.

Brodal, P., Bjaalie, J.G., Aas, J.E., 1991. Organization of cingulo-ponto-cerebellar connections in the cat. Anat. Embryol. 184, $245-254$.

Burghardt, N.S., Sullivan, G.M., McEwen, B.S., Gorman, J.M., LeDoux, J.E., 2004. The selective serotonin reuptake inhibitor citalopram increases fear after acute treatment but reduces fear with chronic treatment: A comparison with tianeptine. Biol. Psychiat. 55, 1171-1178.

Cantalupo, C., Hopkins, W., 2010. The cerebellum and its contribution to complex tasks in higher primates: A comparative perspective. Cortex. 46, 821-830.

Cavdar, S., Onat, F.Y., Yananli, H.R., Sehirli, U.S., Tulay, C., Saka, E., Gürdal, E., 2002. Cerebellar connections to the rostral reticular nucleus of the thalamus in the rat. J. Anat. 201, 485-491.

de Ribaupierre, S., Ryser, C., Villemure, J.G., Clarke, S., 2008. Cerebellar lesions: Is there a lateralisation effect on memory deficits? Acta Neurochir. 150, 545-550.

Desbonnet, L., Temel, Y., Visser-Vandewalle, V., Blokland, A., Hornikx, V., Steinbusch, H.W., 2004. Premature responding following bilateral stimulation of the rat subthalamic nucleus is amplitude and frequency dependent. Brain Res. 1008, 198-204.

Dietrichs, E., Haines, D.E., Røste, G.K., Røste, L.S., 1994. Hypothalamocerebellar and cerebellohypothalamic projections-circuits for regulating nonsomatic cerebellar activity? Histol. Histopathol. 9, 603-614.

Duan, X.Q., Wu, S.L., Li, T., Liang, J.C., Qiou, J.Y., Rao, Z.R., Ju, G., 1999. Expression and significance of three types of Fos-immunoreactive cells after gamma knife irradiation of the forebrain in the rat. Neurosci. Res. 33, 99-104.

Fine, E.J., Ionita, C.C., Lohr, L., 2002. The history of the development of the cerebellar examination. Semin. Neurol. 22, 375-384.

Glickstein, M., Strata, P., Voogd, J., 2009. Cerebellum: History. Neuroscience. 162, 549-559.

Leiner, H.C., Leiner, A.L., Dow, R.S., 1986. Does the cerebellum contribute to mental skills? Behav. Neurosci. 100, 443-454.

Leiner, H.C., Leiner, A.L., Dow, R.S., 1993. Cognitive and language functions of the human cerebellum. Trends Neurosci. 16, $444-447$.

Lim, L.W., Blokland, A., Tan, S., Vlamings, R., Sesia, T., Aziz-Mohammadi, M., Visser-Vandewalle, V., Steinbusch, H.W., Schruers, K., Temel, Y., 2010. Attenuation of fear-like response by escitalopram treatment after electrical stimulation of the midbrain dorsolateral periaqueductal gray. Exp. Neurol. 226, 293-300.

Lim, L.W., Blokland, A., Visser-Vandewalle, V., Vlamings, R., Sesia, T., Steinbusch, H., Schruers, K., Griez, E., Temel, Y., 2008. High-frequency stimulation of the dorsolateral periaqueductal gray and ventromedial hypothalamus fails to inhibit panic-like behaviour. Behav. Brain Res. 193, 197-203.

Lim, L.W., Temel, Y., Visser-Vandewalle, V., Steinbusch, H., Schruers, K., Hameleers, R., Esquivel, G., Griez, E., Blokland, A., 2008. Effect of buspirone on the behavioral regulation of rats in low versus high anxiety conditions. Arzneimittelforschung. 58, 269-276.

Lino-de-Oliveira, C., de Oliveira, R.M., Pádua Carobrez, A., de Lima, T.C., del Bel, E.A., Guimarães, F.S., 2006. Antidepressant treatment reduces Fos-like immunoreactivity induced by swim stress in different columns of the periaqueductal gray matter. Brain Res. Bull. 70, 414421.

Manto, M., 2008. The cerebellum, cerebellar disorders, and cerebellar research--two centuries of discoveries. Cerebellum. 7, 505-516.

Marien, P., Engelborghs, S., De Deyn, P.P., 2001. Cerebellar neurocognition: A new avenue. Acta Neurol. Belg. 101, 96-109.

Middleton,, F.A., Strick, P.L., 2000. Basal ganglia and cerebellar loops: Motor and cognitive circuits. Brain Res. Rev. 31, $236-250$.

Middleton, F.A., Strick, P.L., 2001. Cerebellar projections to the prefrontal cortex of the primate. J. Neurosci. 21, 700-712.

Moers-Hornikx, V.M., Sesia, T., Basar, K., Lim, L.W., Hoogland, G., Steinbusch, H.W., Gavilanes, D.A., Temel, Y., Vles, J.S., 2009. Cerebellar nuclei are involved in impulsive behaviour. Behav. Brain Res. 203, 256-263.

Moers-Hornikx, V.M., Vles, J.S., Lim, L.W., Ayyildiz, M., Kaplan, S., Gavilanes, A.W., Hoogland, G., Steinbusch, H.W., Temel, Y., 2011. Periaqueductal grey stimulation induced panic-like behaviour is accompanied by deactivation of the deep cerebellar nuclei. Cerebellum. 10, 61-69.

Moers-Hornikx, V.M., Vles, J.S., Tan, S.K., Cox, K., Hoogland, G., Steinbusch, W.M., Temel, Y., 2011. Cerebellar nuclei are activated by highfrequency stimulation of the subthalamic nucleus. Neurosci. Lett. 496, 111-115.

Paulesu, E., Frith, C.D., Frackowiak, R.S.,1993. The neural correlates of the verbal component of working memory. Nature. 362, $342-345$.

Pearce, J.M., 2009. Marie-Jean-Pierre Flourens (1794-1867) and cortical localization. Eur. Neurol. 61, 311-314.

Sacchetti, B., Scelfo, B., Strata, P., 2005. The cerebellum: synaptic changes and fear conditioning. Neuroscientist. 11, $217-227$.

Sacchetti, B., Scelfo, B., Strata, P., 2009. Cerebellum and emotional behavior. Neuroscience. 162, 756-762.

Sato, H, Skelin, I., Debonnel, G., Diksic, M., 2008. Chronic buspirone treatment normalizes open field behavior in olfactory bulbectomized rats: Assessment with a quantitative autoradiographic evaluation of the 5-HT1A binding sites. Brain Res. Bull. 75, 545-555.

Schmahmann, J.D., 1996. From movement to thought: Anatomic substrates of the cerebellar contribution to cognitive processing. Hum. Brain Mapp. 4, 174-198.

Schmahmann, J.D., 2010. The role of the cerebellum in cognition and emotion: Personal reflections since 1982 on the dysmetria of thought hypothesis, and its historical evolution from theory to therapy. Neuropsychol. Rev. 20, 236-260.

Schmahmann, J.D., Pandya, D.N., 1991. Projections to the basis pontis from the superior temporal sulcus and superior temporal region in the rhesus monkey. J. Comp. Neurol. 308, 224-248.

Schmahmann, J.D., Pandya, D.N., 1993. Prelunate, occipitotemporal, and parahippocampal projections to the basis pontis in rhesus monkey. J. Comp. Neurol. 337, 94-112. 
Schmahmann, J.D., Sherman, J.C., 1998. The cerebellar cognitive affective syndrome. Brain. 121, 561-579.

Smith, M.A., Banerjee, S., Gold, P.W., Glowa, J., 1992. Induction of c-fos mRNA in rat brain by conditioned and unconditioned stressors. Brain Res. 578, 135-141.

Snider, R.S., Maiti, A., 1976. Cerebellar contributions to the Papez circuit. J. Neurosci. Res. 2, 133-146.

Tan, S.Kh., Vlamings, R., Lim, L., Sesia, T., Janssen, M.L., Steinbusch, H.W., Visser-Vandewalle, V., Temel, Y., 2010. Experimental deep brain stimulation in animal models. Neurosurgery. 67, 1073-1079.

Temel, Y., Boothman, L.J., Blokland, A., Magill, P.J., Steinbusch, H.W., Visser-Vandewalle, V., Sharp, T., 2007. Inhibition of 5-HT neuron activity and induction of depressive-like behavior by high-frequency stimulation of the subthalamic nucleus. P. Natl. Acad. Sci. USA. 104, 17087-17092.

Turner, B.M., Paradiso, S., Marvel, C.L., Pierson, R., Boles Ponto, L.L., Hichwa, R.D., Robinson, R.G., 2007. The cerebellum and emotional experience. Neuropsychologia. 45, 1331-1341.

Watson, G.P.C., 1998. The rat brain in stereotaxic coordinates, Academic press. 61-68

Wells, E.M., Walsh, K.S., Khademian, Z.P., Keating, R.F., Packer, R.J., 2008. The cerebellar mutism syndrome and its relation to cerebellar cognitive function and the cerebellar cognitive affective disorder. Dev. Disabil. Res. Rev. 14, 221-228.

Yamamoto, T., Yoshida, K., Yoshikawa, H., Kishimoto, Y., Oka, H.,1992. The medial dorsal nucleus is one of the thalamic relays of the cerebellocerebral responses to the frontal association cortex in the monkey: Horseradish peroxidase and fluorescent dye double staining study. Brain Res. 579, 315-320. 